\title{
Biological impact of geometric uncertainties: what margin is needed for intra-hepatic tumors?
}

\author{
Hsiang-Chi Kuo*1,2, Wen-Shan Liu³, Andrew Wu1,4, Dennis Mah¹, Keh-Shih Chuang², Linda Hong ${ }^{1}$, Ravi Yaparpalvi ${ }^{1}$, \\ Chandan Guha' and Shalom Kalnicki ${ }^{1}$
}

\begin{abstract}
Background: To evaluate and compare the biological impact on different proposed margin recipes for the same geometric uncertainties for intra-hepatic tumors with different tumor cell types or clinical stages.

Method: Three different margin recipes based on tumor motion were applied to sixteen IMRT plans with a total of twenty two intra-hepatic tumors. One recipe used the full amplitude of motion measured from patients to generate margins. A second used $70 \%$ of the full amplitude of motion, while the third had no margin for motion. The biological effects of geometric uncertainty in these three situations were evaluated with Equivalent Uniform Doses (EUD) for various survival fractions at $2 \mathrm{~Gy}_{(}\left(\mathrm{SF}_{2}\right)$.

Results: There was no significant difference in the biological impact between the full motion margin and the 70\% motion margin. Also, there was no significant difference between different tumor cell types. When the margin for motion was eliminated, the difference of the biological impact was significant among different cell types due to geometric uncertainties. Elimination of the motion margin requires dose escalation to compensate for the biological dose reduction due to the geometric misses during treatment.
\end{abstract}

Conclusions: Both patient-based margins of full motion and of $70 \%$ motion are sufficient to prevent serious dosimetric error. Clinical implementation of margin reduction should consider the tumor sensitivity to radiation.

\section{Background}

Primary hepatocellular carcinoma (HCC) and liver metastases are common in East Asia and Africa. The volume of liver cancer patients in the United States increases each year [1]. Due to the poor tolerance of the whole liver to radiation, radiation therapy (RT) has conventionally played a very limited role in treating liver cancer. Recently, advanced RT techniques (3D conformal \& stereotactic radiotherapy) have been applied to unresectable focal intrahepatic cancer to improve the local control rate without serious radiation-induced liver disease (RILD) [2,3]. Michigan's group [2] has showed that HCC treatment with RT is promising. In particular, the response rate, measured by the shrinkage of the tumor volume, could be as high as $90 \%$. In 2002, HC Park et al [4] found the response rates of $\mathrm{HCC}$ were $29.2 \%, 68.6 \%$, and $77.1 \%$ for doses $40 \mathrm{~Gy}, 40-50 \mathrm{~Gy}$, and $50 \mathrm{~Gy}$, respectively (corre-

\footnotetext{
* Correspondence: hskuo@montefiore.org

1 Department of Radiation Oncology, Montefiore Medical Center, USA Full list of author information is available at the end of the article
}

sponding to a BED of 47.2 Gy, 47.2-59 Gy, and 59 Gy, respectively). Another group [5] found that the response rates were $46.7 \%$ in biological equivalent dose (BED) $<50$ Gy and $72.8 \%$ in BED > 50 Gy. For the treatment of HCC with portal vein thrombosis (PVT), two other groups [6,7] also showed a dose dependence of the local tumor response. These clinical results [2-6] \&[7] reveal that intra-hepatic tumor radiation response is dose dependent regardless of the presence of PVT.

Another potential biologic marker of local recurrence after radiotherapy is the intrinsic tumor radiosensitivity. Using $\mathrm{SF}_{2}$ (surviving fraction of tumor cell colony at $2 \mathrm{~Gy}$ ) as an end point for intrinsic radiosensitivity, some clinical studies have evaluated the correlation of $\mathrm{SF}_{2}$ with clinical stage as an independent prognostic factor of local tumor control [8-10]. These studies demonstrated a close association of $\mathrm{SF}_{2}$ with recurrence of cervix, and head and neck tumors, but not glioblastomas. The mechanism of radiosensitivity of hepatocarcinoma cells after radiotherapy is not well understood. However, laboratory studies 
[11] have confirmed that $\mathrm{SF}_{2}$ was significantly correlated with the hepatic carcinoma cell radiosensitivity. Since radiosensitivity is an important factor influencing the prognosis of radiotherapy treatment, it is important to consider the radiation response for different clinical stages and the tumor cell types in the treatment of intrahepatic tumors.

An intra-hepatic tumor is a lesion situated within the abdomen, which has great geometric uncertainty due to respiratory motion $(1 \sim 2.5 \mathrm{~cm})$ [12] and daily setup variations $(0.5 \sim 1 \mathrm{~cm})$. These uncertainties may affect the radiotherapy treatment outcome especially for Intensity Modulated Radiotherapy (IMRT) delivery. The effects of organ motion on dose delivery by dynamic multi-leaf collimators (DMLC) have been studied extensively [13-15]. These results showed that the interplay between MLC leaf motion and organ motion did not influence the expected dose to the moving organ in highly fractionated IMRT delivery (approximately thirty fractions). They also found that a one $\mathrm{cm}$ motion margin is clinically acceptable in terms of fluence distortion and CTV (clinical target volume) coverage. Investigations of the influence of setup uncertainty on target coverage have focused on less mobile targets such as on head and neck and prostate cancer $[16,17]$. Recently Balter [18] evaluated the setup uncertainties on treatment plans for focal liver tumors and found the change of the effective normal liver volume difference was within 3\%.

Geometric uncertainties are traditionally overcome by adding adequate margin to CTV to ensure target dose coverage and normal tissue sparing. ICRU (International Commission on Radiation Units and Measurements) report 62 [19] introduced the concept of an internal margin (IM) to take into account variations in size, shape, and position of the CTV in reference to the patient's anatomical reference points and also the concept of a setup margin (SM) to take into account all uncertainties in patientbeam positioning in reference to the treatment machine coordinate system. Report 62 suggests, instead of adding the internal margin and the setup margin linearly, a compromise has to be sought. The majority of the margin schemes are aimed at maintaining a minimum dose (e.g. $95 \%$ of the prescribed dose) to the CTV for a majority (e. g. 90\%) of a patient population or a group of test plans $[20,21]$. For liver, the uncertainty attributed to respiratory motion is large compared to other setup errors and should be considered separately. Mckenzie et al [22] proposed a full respiratory motion amplitude $(A)$ be added on top of other errors. Ten Haken et al [23] proposed the elimination of the respiratory margin while escalating the dose to an amount with the same normal tissue complication probability (NTCP) of normal liver. Van Herk et al [21] proposed a $0.7 A$ margin for motion amplitudes larger than one $\mathrm{cm}$. These studies compared the geomet- rical impact for fractionated treatment with or without biological model. Molinelli et al [24] compared different margin protocols with $0 \mathrm{~mm}, 5 \mathrm{~mm}$, and $10 \mathrm{~mm}$ in either radial or cranial-caudal directions for SBRT treatment of liver with single tumor type of $\mathrm{SF}_{2}=0.5$.

Table 1 summarizes the margin recopies and purposes of these studies. None of these studies correlate the size of margin with different clinical stage or the cell types of different radiosensitivity. Since the clinical stage and the radiosensitivity of different cell types are important prognostic factors of the tumor response and tumor control, we hypothesize that the margin defined for intrahepatic tumors should also consider variations in radiosensitivity which would depend upon different tumor cell types and different clinical stages. Here, different proposed margin schemes were compared for the dose smearing results on the targets with the same geometric uncertainties. EUD is an approach which calculates a uniform dose value from a non-uniform dose distribution that would result in the same biological effects (the survival of the same number of clonogens) in both. The non-uniform dose distributions after the dose smearing were converted into EUD at different $\mathrm{SF}_{2}$ values to evaluate the biological impact of geometric uncertainties. After correlating $\mathrm{SF}_{2}$ of the $\mathrm{HCC}$ tumor cell with different cell types and $\mathrm{HCC}$ stage, the biological impact of the geometric uncertainty was taken into account to guide the clinical decision of creating margins for different stages of intra-hepatic tumors.

\section{Methods}

\section{Data acquisition}

Eight patients with unresectable tumors within the liver were planned with non-gated and gated techniques (Varian RPM system). The details of the RPM system have been described in detail previously [25]. Briefly, a small plastic box with infra-red (IR) reflective markers is placed on the patient. An array of IR LEDs illuminates the box while a camera monitors the displacement of the box due to patient breathing. The respiratory motion for each patient was recorded through the observation of the diaphragm movement under the fluoroscopy with the RPM system installed on a Ximatron simulator (Varian Medical Systems, Palo Alto, USA). The diaphragm's movements during a $100 \%$ amplitude window (peak to trough) and another 50\% amplitude window (mid-peak to trough) were measured in order to expand the CTV and generate a PTV (planning target volume). The maximum motion extent measurements with the $50 \%$ amplitude window were made in order to increase the number of analyzed plans in this study consisting of smaller extents of motion. The trajectories of the movements were also plotted as a motion distribution of $f_{p}(r)$ where $r$ is the displacement of the diaphragm relative to the end exhalation 
Table 1: Summary of motion margin recipes and the study designs.

\begin{tabular}{|c|c|c|c|c|}
\hline Author & Recipe & Biological model & & Purpose \\
\hline Haken et al. 1997 & $\mathrm{OA}$ & $\begin{array}{l}\mathrm{NTCP} \text { (Lyman model) } \\
\mathrm{TD}_{50}=45 \mathrm{~Gy}, \mathrm{~m}=0.15, \mathrm{~m}=0.69 ; \\
\mathrm{TCP} \text { (Simple logistic function) } \\
\mathrm{D}_{50}=60 \mathrm{~Gy}, \mathrm{k}=4\end{array}$ & & $\begin{array}{l}\text { To investigate potential benefits of } \\
\text { eliminating motion margin through liver } \\
\text { tumor treated with conformal therapy }\end{array}$ \\
\hline McKenzie et al. 2000 & A & No & & $\begin{array}{l}\text { How should motion margin be } \\
\text { combined with other margins around CTV }\end{array}$ \\
\hline Van Herk et al. 2003 & $0.7 \mathrm{~A}$ & $\begin{aligned} B E D & =D+\left(1+\frac{D}{\alpha / \beta}\right) \\
\alpha / \beta & =1 \sim 10\end{aligned}$ & & $\begin{array}{l}\text { To investigate biologic and physical } \\
\text { fractionation effects of random } \\
\text { geometric errors and respiration motion } \\
\text { with Gaussian blurring of the plan dose }\end{array}$ \\
\hline Molinelli et al. 2008 & $\begin{array}{l}0 \\
5 \mathrm{~mm} \\
10 \mathrm{~mm}\end{array}$ & $\begin{array}{l}\operatorname{EUD}\left(\mathrm{SF}_{2}=0.5\right) \\
\operatorname{gEUD}(a=-20)\end{array}$ & & $\begin{array}{l}\text { To quantify the potential benefits of } \\
\text { CTV-to-PTV margin reduction for SBRT of } \\
\text { liver tumor }\end{array}$ \\
\hline \multirow[t]{2}{*}{ This study } & $\begin{array}{l}1 \mathrm{~A} \\
0.7 \mathrm{~A} \\
0 \mathrm{~A}\end{array}$ & $E U D=D_{r e f} * \ln \left[\frac{1}{N} \sum_{i}^{N}\left(S F_{2}\right)^{D_{i} / D_{r e f}}\right.$ & $/ \ln \left(S F_{2}\right)$ & $\begin{array}{l}\text { To investigate adequate margin for } \\
\text { different clinical stage of liver tumor } \\
\text { treated with fractionation IMRT }\end{array}$ \\
\hline & & $\mathrm{D}_{\text {ref }}=2 \mathrm{~Gy} ; \mathrm{SF}_{2}=0.3,0.5,0.7$ & & \\
\hline
\end{tabular}

position p. A total of $11 \mathrm{CTVs}$ from eight patients (three of the patients have two lesions) were contoured from CT in this study. Each patient was planned using both the full and half amplitudes of motion such that sixteen plans were generated in total.

The study with motion from fluoroscopy assumed that the liver was dragged by the diaphragm along the cranialcaudal direction without any changes in size and shape. CT images with $5 \mathrm{~mm}$ thickness used for treatment planning were acquired at end exhalation. Calculations were performed at a $2.5 \mathrm{~mm}$ grid size. The objectives of the planning were to minimize the mean dose to the normal tissue with at least $95 \%$ of the PTV covered with 50 Gy.

In contrast to the motion study without considering the deformation, a single patient was planned with 4DCT images for comparison. The 4DCT image set was acquired using a GE (General Electric Medical system, Buckinghamshire, UK) Lightspeed 16-slice CT scanner with Varian's RPM system. Images were scanned at 0.5 seconds per revolution and a 4DCT protocol developed by Rietzel et al [26] was followed. The reconstructed CT images had a voxel size of $2.5 \mathrm{~mm}$ in the superior-inferior (SI) direction, and $1 \mathrm{~mm}$ in the anterior-posterior (AP) and right-left (RL) directions. After the 10 phase CT images were sorted and organ motion was studied in the GE Advantage4D workstation, the 10 phase CT images were exported to Varian Eclipse (version 8.6) workstation. A 4D planning scheme was performed which incorporated the patient's motion model in 3D

\section{Margin design}

The planning target volume (PTV) was generated according to recommendations of ICRU Report 62 with internal margins (IM) and setup margins (SM). The CTV to PTV expansion was calculated by the root of the sum of squares of IM and SM. Brock et al [27] have investigated intra-hepatic lesions including an isotropic $5 \mathrm{~mm}$ expansion for setup error, an inferior margin for the patientspecific range of the diaphragm movement due to breathing, and an addition $3 \mathrm{~mm}$ superior expansion for reproducibility of the exhale state [28]. Based upon the margin design in Brock's definition of PTV, we compared the effect of geometric uncertainty with different margin recipes. Conventionally, we would select a PTV margin around a CTV. The aim of this study was to obtain information on the discrepancy between the static and motion blurred IMRT dose distribution resulting from geometrical error. To reduce the number of plan calculations, we considered the reverse approach, which is to keep the PTV constant, but change the size of the CTV (figure 1). Hence, we introduce the $\mathrm{CTV}, \mathrm{CTV}_{\mathrm{a}}$ and $\mathrm{CTV}_{\mathrm{b}}$ described as follows.

1) The lesion on $C T$ images enhanced with contrast was defined as the CTV. The full diaphragmatic motion (the amplitude of respiration) and the PTV expansion method described above were applied for the CTV margin.

2) To comply with Van Herk's $70 \%$ motion margin recipe, the original CTVs were expanded $0.3 \mathrm{~A}$ caudally as $\mathrm{CTV}_{\mathrm{a}}$ such that $\mathrm{CTV}_{\mathrm{a}}$ could construct the same PTV from an expansion of Van Herk's margin.

3) We approximate elimination of the motion margin by creating $\mathrm{CTV}_{\mathrm{b}}$ with the addition of another $0.5 \mathrm{~cm}$ margin to $\mathrm{CTV}_{\mathrm{a}}$ (i.e. one CT slice, due to CT thickness, the exact number of full motion elimination was 


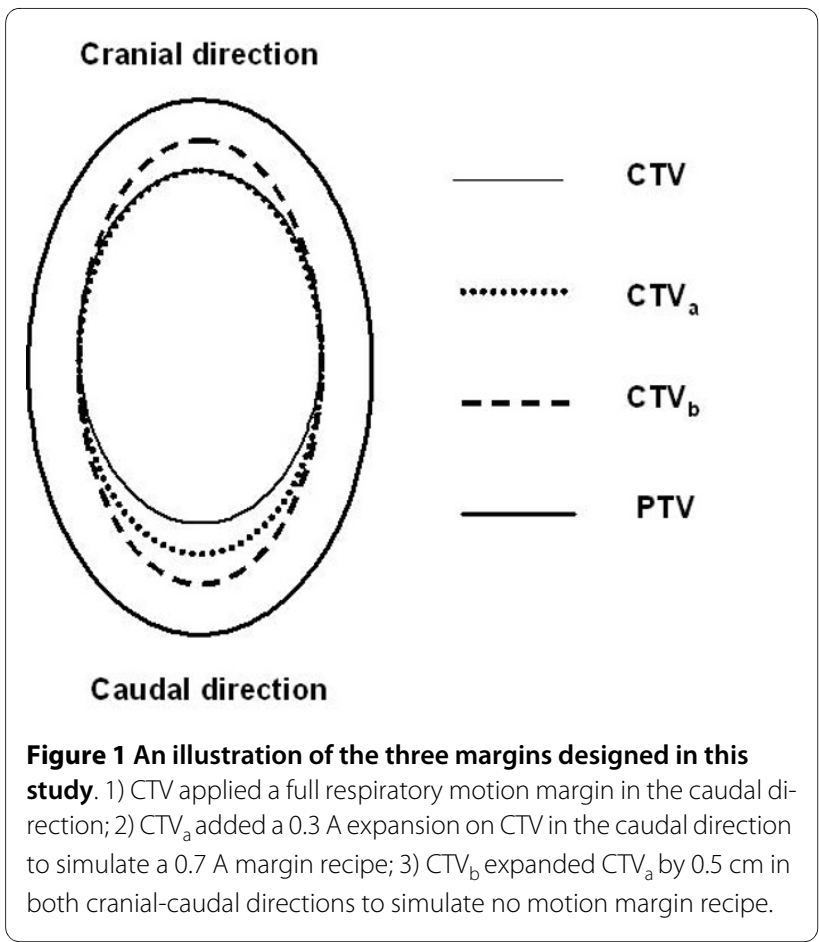

difficult to reached) in both cranial and caudal directions. This expansion simulates a further margin reduction in the cranial-caudal direction from the PTV.

With the above approximations, all three CTVs are expanded to the same PTV. CTV is the original clinical target volume, $\mathrm{CTV}_{\mathrm{a}}$ is close to a clinical target volume with a reduced margin of $0.7 \mathrm{~A}$, and $\mathrm{CTV}_{\mathrm{b}}$ is close to a clinical target volume excluding a motion margin but including a setup margin.

\section{Incorporation of geometric errors: Two step convolution}

Convolution is a mathematical model for combining two functions into a third function. It is an established procedure for converting an input object (function 1, either an image, fluence, or dose distribution) in motion (function 2 , either a filter, motion distribution, or probability distribution) into an output object (function 3, same attribute as function 1) with the motion smearing effect. When the input object is an image, motion will blur the image in the same way that a moving object is blurred in photography. When the input object is a fluence or dose distribution, motion will blur the fluence or dose. Convolution incorporates the motion function to output an object which simulates the blurred image, fluence, or dose distribution. Motion smearing of the fluence or dose distribution causes the broadening of the penumbra and the degradation of the target coverage. This study applied a two-step convolution to simulate the dose received by the patient.
The first step convolved the fluence created by the MLC with the patient's moving diaphragm distribution to generate an effective fluence. The second step convolved the dose matrix calculated from the effective fluence with a Gaussian distribution representing the setup error. The first step considered the inhomogeneity of the body. The second step assumed that the body is homogeneous. The effective fluence method for the dose distortion by patient motion has been validated in our previous study [15].

Sixteen IMRT plans were created for PTV and PTVg (maximum motion extent taken from the 50\% motion window) of eight liver patients for a total of 24 CTVs. The dose prescription was 50 Gy to the PTV with 2 Gy per fraction (25 fractions) for each plan. The dynamic MLC motion files from the planning system (Varian, Cadplan, Varian Medical Systems, Palo Alto, USA) were then convolved with the motion function [11] to obtain the effective fluence

$$
\Phi p=\int I\left(x_{r}^{k}(t)-\chi\right) I\left(\chi-x_{l}^{k}(t)\right) d t
$$

where $x^{k}{ }_{r}(t)$ and $x^{k}{ }_{l}(t)$ denote the positions of the right and left leaves (relative to the iso-center), respectively, of the $k$ th leaf pair. $I$ is the intensity distribution generated from the leaf motion (which is in perpendicular direction to the diaphragm movement in this study). For an arbitrary point in the organ, $\mathrm{p}$, if the fluence has no distortion due to the motion of an organ, $X$ is constant. In the calculation of the fluence following distortion due to organ motion, $X$ is substituted for the pre-known motion function,

$$
\chi=f_{p}\left(t+t_{0} ; \zeta, A\right)
$$

Where $f_{p}()$ is the motion function for point $\mathrm{p}$; $\zeta$ is the period; $A$ is the motion amplitude; $t$ is the beam-on-time, and $t_{o}$ is the initial phase. In the calculation of the convolution, the specific patient motion trajectory distribution $\mathrm{f}_{\mathrm{p}}()$ acquired in previous section was incorporated into equation (1). During the convolution process, the initial phase was randomly sampled 25 times to simulate 25 different treatments. The static fluences and the effective fluences were incorporated back to the planning system for forward dose calculation [29]. The dose distributions of static fluences were considered as static plans without motion and the dose distributions of effective fluences were considered as plans incorporating motion.

After the dose distribution was obtained from the effective fluence, a second convolution was performed by convolving the dose matrix with a three dimensional Gaussian probability distribution function [30,31], 


$$
D_{m r}(R)=\iiint_{R^{\prime}(x, y, z)} D_{m}\left(R^{\prime}\right) \bullet P D F_{G}\left(R-R^{\prime}\right) d R^{\prime}
$$

where $D_{m}$ is the dose matrix incorporating with motion effect, $D_{m r}(R)$ is the expected dose distribution (at $R(x, y, z)$ ) blurred by the random setup uncertainty. The random setup uncertainty is described by an isotropic 3D Gaussian distribution $\left(\mathrm{PDF}_{\mathrm{G}}\right)$ with a standard deviation of $0.5 \mathrm{~cm}$ in the anterior-posterior (AP), lateral (LAT), and caudal-cranial (CC) directions [31].

\section{Systematic Error}

Systematic error might occur during the image acquisition or treatment execution. To compare the effects of random and systematic errors, an arbitrary offset of 0.5 $\mathrm{cm}$ (in contrast to random error and the criteria of an acceptable tolerance in practice) was applied at isocenter for the effective fluence before the final dose calculations were done at 1 ) caudal (-z) direction; 2$)$ cranial (+z) direction; 3) $0.35 \mathrm{~cm}$ at both of the right lateral (x) and anterior (y) direction. The latter represents the expansion in both $\mathrm{x}$ and $\mathrm{y}$ directions such that $\left(\mathrm{x}^{2}+\mathrm{y}^{2}\right)^{1 / 2}=0.5$.

\section{Plan Evaluation}

EUD as defined by Niemierko [32] is given by,.

$$
\operatorname{EUD}(G y)=D_{\text {ref }} * \ln \left[\frac{1}{N} \sum_{i}^{N}\left(S F_{2}\right)^{D_{i} / D_{\text {ref }}}\right] / \ln \left(S F_{2}\right)
$$

Equation 4 was used to compare the effect of geometric uncertainties on EUD by varying the parameter $\mathrm{SF}_{2}$ over a range of values $(0.3,0.5$, and 0.7$)$ to represent very radiosensitive, medium radiosensitive, and radioresistant tumor cell types, respectively. $\mathrm{D}_{\text {ref }}$, the reference fraction dose, was $2 \mathrm{~Gy}$ in this study in conjunction with $\mathrm{SF}_{2}$.

The static plans were compared with plans incorporating geometric uncertainties for CTV, CTV, $\mathrm{CTV}_{\mathrm{b}}$ and PTV in terms of cold spots, as defined by: 1) the dose encompassing $99 \%$ of the volume $\left.\left(\mathrm{D}_{99 \% \mathrm{~V}}\right), 2\right)$ the fraction of the target volume with dose $10 \%\left(\mathrm{~V}_{90 \% \mathrm{D}}\right)$, and $5 \%$ lower $\left(\mathrm{V}_{95 \% \mathrm{D}}\right)$ than the prescription dose. To compare the biological effects, DVHs were converted to equivalent uniform dose (EUD), using equation (4), then the impact of geometric uncertainty was calculated as the percentage of dose error:

$$
\%(\Delta E U D)=100 \% * \frac{\left(E U D_{G U}-E U D_{\text {plan }}\right)}{E U D_{\text {plan }}}
$$

where $\mathrm{EUD}_{\mathrm{GU}}$, and $\mathrm{EUD}_{\text {plan }}$ are the EUD with and without geometric uncertainty, respectively.

\section{Statistical Analysis}

The plan evaluation results at the above end points $\left(\mathrm{D}_{99 \% \mathrm{~V}}, \mathrm{~V}_{90 \% \mathrm{D}}, \mathrm{V}_{95 \% \mathrm{D}}, \%(\Delta \mathrm{EUD})\right.$ at $\mathrm{SF}_{2}=0.3,0.5$, and 0.7$)$ for different margins (different CTVs) were analyzed using SAS software (SAS institute inc., release 8.1). The statistical significance of the difference between these end points was determined using a two sided paired sample $t$ test, where the end points of the CTVs are paired by patient. Differences of the results were reported to be significant at $\mathrm{p}<0.05$.

\section{Comparison with 4D study}

A study incorporated 4D CT data which accounted for the organ deformation during respiration was compared with the study above. The details of the $4 \mathrm{D}$ method were mentioned in a separate report [33]. In brief, a 4D plan was done by warping the static dose distribution of different phases of CT images with a 3D deformation map such that the overall dose at each tissue voxel was accumulated at the reference CT image. The 3D deformation map was generated after deformable registration registered 4D CT images into the reference CT image. A diffeormorphic registration algorithm was built upon ITK's (Insight Segmentation and Registration Toolkit) environment to perform the deformable registration. Another in-house program was developed to synchronize dynamic MLC segments with respiration phases such that static dose distribution of different phases can be obtained from the sorted synchronized DMLC segments. To account for the random set up uncertainty, a convolution similar to equation (3) was applied at the static dose distribution of each respiration phase before the dose distribution was warped with the deformation map. After the dose warping, the deformed dose distributions from each respiratory phase were summed together as the simulated $4 \mathrm{D}$ dose distribution.

\section{Results}

The CTV volumes ranged between 7 and $206 \mathrm{cc}$ (mean 88 cc) and the motion amplitudes ranged from 0.9 to $1.9 \mathrm{~cm}$ $($ mean $1.33 \mathrm{~cm})$. The three different CTVs $\left(\mathrm{CTV}, \mathrm{CTV}_{\mathrm{a}}\right.$ and $\mathrm{CTV}_{\mathrm{b}}$ ) and their volumes with PTV margins are listed in table 2. Figure 2 shows dose volume histograms (DVH) averaged over the patient population. The solid lines refer to the original plan and the dashed lines refer to the plan with the effects of geometric uncertainties due to organ motion and random setup error. Also shown are the different data points of intrest $\left(D_{99 \%}, V_{90 \% \mathrm{D}}\right.$, and $\left.\mathrm{V}_{95 \% \mathrm{D}}\right)$. The error bars on these points indicate the range over the patient population. Since the margin for the CTV and CTV $_{\mathrm{a}}$ are sufficient to overcome the effect of geometric errors, their DVHs with and without motion are effectively identical. The mean $\pm 1 \mathrm{SD}$ of the $\mathrm{D}_{99 \% \mathrm{~V}}$, 
Table 2: Patient data with amplitudes, targets and the margins in this study (P3, P5, and P6 have two lesions).

\begin{tabular}{|c|c|c|c|c|c|}
\hline \multirow[t]{2}{*}{ patient } & \multirow{2}{*}{$\begin{array}{c}\text { Amplitude } \\
\mathrm{cm}^{*}\end{array}$} & \multicolumn{4}{|c|}{ Volume (cc) } \\
\hline & & CTV & $\mathrm{CTV}_{\mathrm{a}}$ & $\mathrm{CTV}_{\mathrm{b}}$ & PTV \\
\hline \multirow[t]{2}{*}{ P1 } & 1.4 & 89 & 121 & 161 & 213 \\
\hline & 0.7 & & & & 175 \\
\hline \multirow[t]{2}{*}{ P2 } & 1.7 & 206 & 266 & 344 & 439 \\
\hline & 0.9 & & & & 352 \\
\hline \multirow[t]{2}{*}{ P3 } & 1.8 & $89 ; 23$ & $148 ; 44$ & $222 ; 70$ & $291 ; 103$ \\
\hline & 1 & & & & $220 ; 86$ \\
\hline \multirow[t]{2}{*}{ P4 } & 1.9 & 90 & 126 & 191 & 367 \\
\hline & 1 & & & & 314 \\
\hline \multirow[t]{2}{*}{ P5 } & 1.2 & $77 ; 7$ & $107 ; 17$ & $149 ; 31$ & $183 ; 43$ \\
\hline & 0.6 & & & & $152 ; 40$ \\
\hline \multirow[t]{2}{*}{ P6 } & 0.9 & $111 ; 42$ & $139 ; 57$ & $184 ; 77$ & $250 ; 117$ \\
\hline & 0.5 & & & & $214 ; 111$ \\
\hline \multirow[t]{2}{*}{ P7 } & 1.1 & 163 & 215 & 287 & 325 \\
\hline & 1.1 & 15 & 28 & 45 & 61 \\
\hline \multirow[t]{2}{*}{ P8 } & 1.6 & 141 & 200 & 284 & 791 \\
\hline & 0.8 & & & & 645 \\
\hline
\end{tabular}

* Each patient was planned with the full and half amplitudes of motion such that sixteen plans were generated in total.

$\mathrm{V}_{90 \% \mathrm{D}}$, and $\mathrm{V}_{95 \% \mathrm{D}}$ for CTV after the geometric uncertainties were $5.2 \pm 3.9$ (Gy), $1.3 \pm 3.7$ (\%), and $7.7 \pm 13.6$ (\%), respectively. Despite a slightly smaller margin than $\mathrm{CTV}_{\mathrm{a}}$, similar results hold for $\mathrm{CTV}_{\mathrm{b}}$, although the coverage for the chosen end points, on average, is lower. Specifically, the mean $\pm 1 \mathrm{SD}$ of the $\mathrm{D}_{99 \% \mathrm{~V}}, \mathrm{~V}_{90 \% \mathrm{D}}$, and $\mathrm{V}_{95 \% \mathrm{D}}$ after the geometric uncertainties were $12.3 \pm 6$ (Gy), $3.2 \pm 4.6$ (\%), and $8.6 \pm 8.6(\%)$, respectively. Finally, in Figure 2(d), the PTV can be considered as a target without any margin for geometric uncertainties. Here, the effect of motion is dramatic and a large portion of the target volume will receive an unacceptably low dose.

Figure 3 shows a box plot of the variation of the biological response $\%(\Delta E U D)$ to the presence of geometric uncertainties for different targets and different survival rates. Both the value and standard deviation of $\%(\Delta E U D)$ increase as the margin decreases. The box plot also shows how $\%(\triangle \mathrm{EUD})$ changes as survival rates (SF2) change. There is no significant change as SF2 increases for both of CTV and CTVa. The geometric uncertainties induced biological effects quantified as \%( $\triangle \mathrm{EUD})$ error, were $1.4 \pm$ $1.9 \%, 1.4 \pm 1.9 \%$, and $1.6 \pm 1.9 \%$ for SF2 of $0.7,0.5$ and 0.3 , respectively. For CTVa, the values of $\%(\triangle \mathrm{EUD})$ were $1.6 \pm$ $1.8 \%, 1.7 \pm 2.0 \%$, and $2.1 \pm 2.3 \%$ for SF2 of $0.7,0.5$ and 0.3 , respectively, which are statistically indistinguishable from CTV. However, for CTVb and PTV, the mean value and variation (as measured by range and standard deviation) both decrease with as SF2 increases. The biological effects were $2.5 \pm 2 \% .1,3.4 \pm 2.4 \%$, and $5.3 \pm 3.5 \%$ for SF2 of $0.7,0.5$ and 0.3 , respectively, for CTVb. The biological impact was largest when there were no motion and setup margins at all (i.e. when CTV $=\mathrm{PTV}$ ).

To establish if the results from figures $2 \& 3$ are statistically significant, a paired sample $t$ test was used to compare the difference between $C T V$ and $\mathrm{CTV}_{\mathrm{a}}$ and the difference between CTV and $\mathrm{CTV}_{\mathrm{b}}$ in terms of $\mathrm{D}_{99 \% \mathrm{~V}}$, $\mathrm{V}_{90 \% \mathrm{D}}, \mathrm{V}_{95 \% \mathrm{D}}, \%(\Delta \mathrm{EUD})$ at $\mathrm{SF}_{2}=0.3,0.5$, and 0.7 . The results of this test are listed in Table 3 . The geometric uncertainty has the same effect on physical and biological DVH for CTV and $\mathrm{CTV}_{\mathrm{a}}$. However, the difference in 

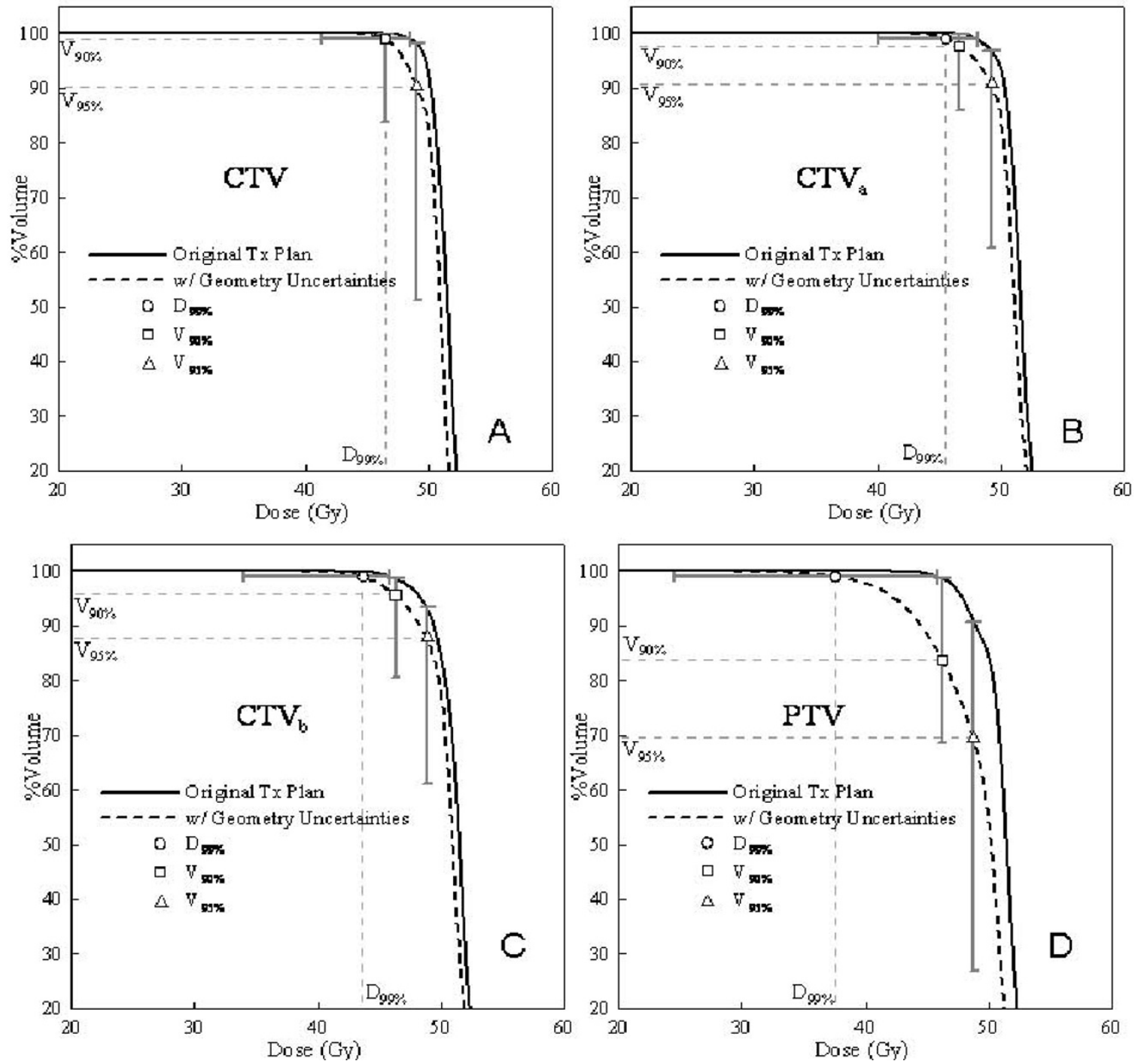

Figure 2 The effects of geometric uncertainty on the DVH of different targets. The DVH curves are the mean curves from different patients in this study. (a), (b), (c) \& (d) correspond to CTV, $C T V_{a}, C T V_{b}$, and PTV, respectively. The o, $\square, \triangle$ symbols show the mean reduction of the $D_{99 \%}, V_{90 \% D}$, and $V_{95 \% \mathrm{D}}$ from the original DVH curves. The bar across the symbols are the range of the data set.

dosimetric impact between $\mathrm{CTV}$ and $\mathrm{CTV}_{\mathrm{b}}$ is significant using most endpoints. We calculated the correlation between the $\%\left(\Delta \mathrm{EUD}_{0.5}\right)$ on $\mathrm{CTV}_{\mathrm{b}}$, motion amplitude, the amount of margin on $\mathrm{CTV}_{\mathrm{b}}$, and the $\%\left(\Delta \mathrm{EUD}_{0.5}\right)$ on CTV. The correlation coefficients between $\mathrm{CTV}_{\mathrm{b}}$ and the rest of the parameters (amplitude, margin, and $\%\left(\Delta \mathrm{EUD}_{0.5}\right)$ on $\mathrm{CTV}_{\mathrm{b}}$ were $0.14,0.58$ and 0.32 , respectively. The strongest correlation between the $\%\left(\Delta \mathrm{EUD}_{0.5}\right)$ on $\mathrm{CTV}_{\mathrm{b}}$ is with the margin size (or more specifically, the lack thereof).

Figure 4 displays the dosimetric error (equation 5) comparisons for both the motion plus random and motion plus systematic errors. We grouped the data into subsets, one for amplitude $>1 \mathrm{~cm}$ and the other $<1 \mathrm{~cm}$. The data are shown for different CTVs resulting from the different margin recipes over a range of tumor cell types. The systematic errors (combined with motion error) displayed in the figure were the worst case of the three simulated center offsets (as systematic errors) in the caudal-cranial and the transverse directions. The results showed that as we combined motion and random error, $\mathrm{CTV}_{\mathrm{b}}$ had a mean $\%(\triangle E \mathrm{EUD})$ reduction of $3 \% \sim 6 \%$, which made no significant difference between the two groups. On the contrary, combined motion with systematic error, for the group of motion amplitude $>1 \mathrm{~cm}$, the $\mathrm{CTV}_{\mathrm{a}}$ had a mean $\%(\Delta \mathrm{EUD})$ reduction of $2 \% \sim 5 \%, \mathrm{CTV}_{\mathrm{b}}$ had a mean 


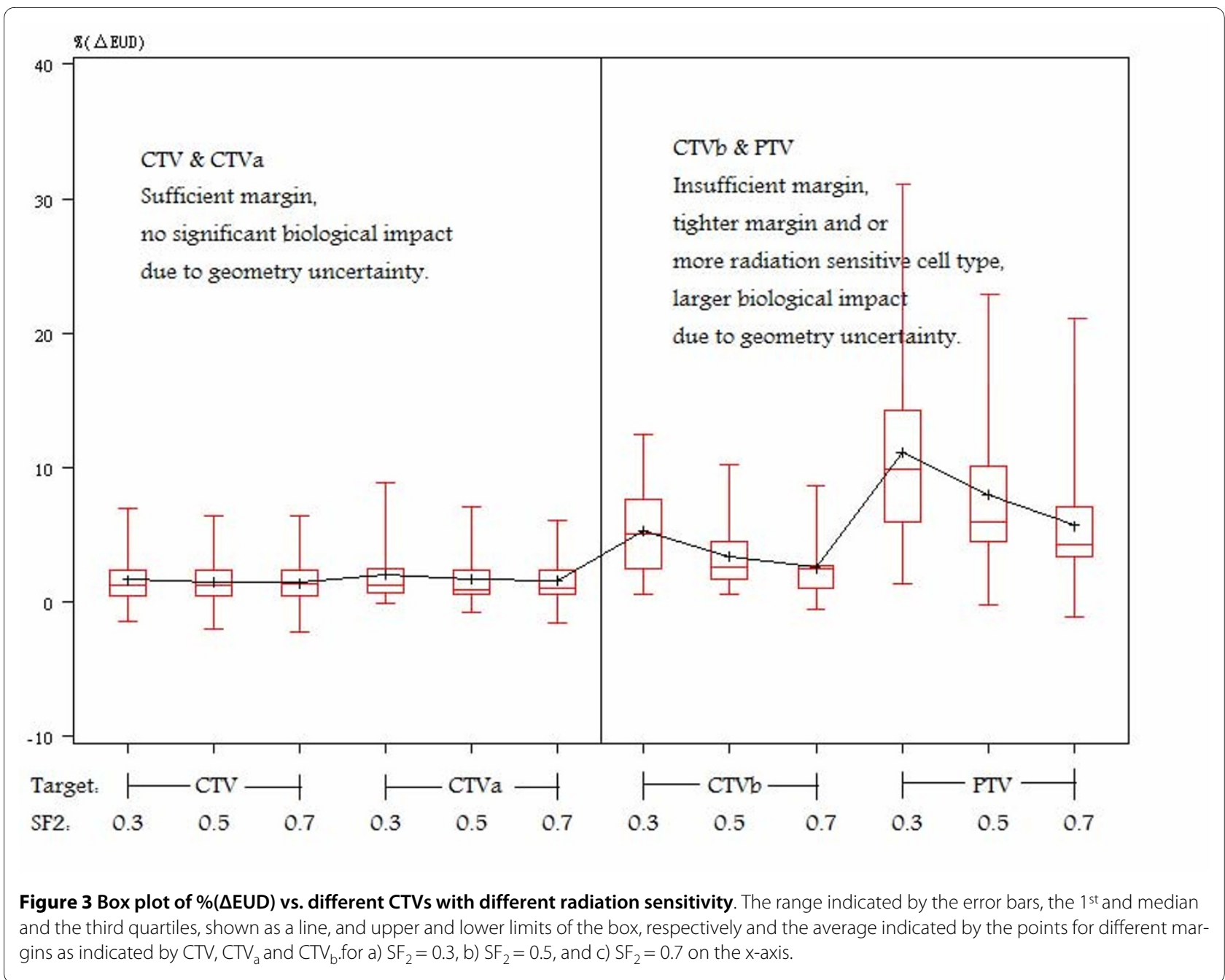

$\%(\Delta \mathrm{EUD})$ reduction of $3 \% \sim 11 \%$. These mean $\%(\Delta \mathrm{EUD})$ errors were twice as high for the group of motion amplitude $<1 \mathrm{~cm}$.

For the case with $4 \mathrm{D}$ data, figure $5 \mathrm{a}$ and $5 \mathrm{~b}$ display the dose profiles without geometry effects ("P"), with set up error ("SM"), with motion error ("IM"), and with geometry impact ("G", combines "SM" \& "IM") in AP ("Y"), and Sup/Inf ("Z") directions, respectively. The motion in the
$\mathrm{RT} / \mathrm{LT}$ direction is not shown since the effect is smaller than the effect in the AP direction. The displacements of the $95 \%$ dose position from planning iso-center due to different geometry uncertainty are summarized in table 4. The degradations (negative value) of the $95 \%$ dose position were $3 \mathrm{~mm}, 2.4 \mathrm{~mm}$, and $12.9 \mathrm{~mm}$ in $\mathrm{RT} / \mathrm{LT}$, AP, and Sup/Inf directions, respectively. Set up uncertainty has effect relatively isotropic in all directions. Respiration

Table 3: The $p$-values of the paired sample t test for $\mathrm{CTV}_{\text {with }} \mathrm{CTV}_{\mathrm{a}}$, and $\mathrm{CTV}$ with $\mathrm{CTV}_{\mathrm{b}}$.

\begin{tabular}{ccc}
\hline & $\mathbf{C T V}_{\mathbf{a}}$ & $\mathbf{C T V}_{\mathbf{b}}$ \\
\hline $\mathrm{D}_{99 \% \mathrm{~V}}$ & 0.049 & $<0.0001$ \\
$\mathrm{~V}_{90 \% \mathrm{D}}$ & 0.1231 & 0.0066 \\
$\mathrm{~V}_{95 \% \mathrm{D}}$ & 0.7049 & 0.6471 \\
$\%\left(\Delta \mathrm{EUD}_{0.7}\right)$ & 0.4017 & 0.0002 \\
$\%\left(\Delta \mathrm{EUD}_{0.5}\right)$ & 0.2526 & $<0.0001$ \\
$\%\left(\Delta \mathrm{EUD}_{0.3}\right)$ & 0.088 & $<0.0001$ \\
\hline
\end{tabular}

Two data sets are the statistically the same if the p-value is larger than 0.05 . 


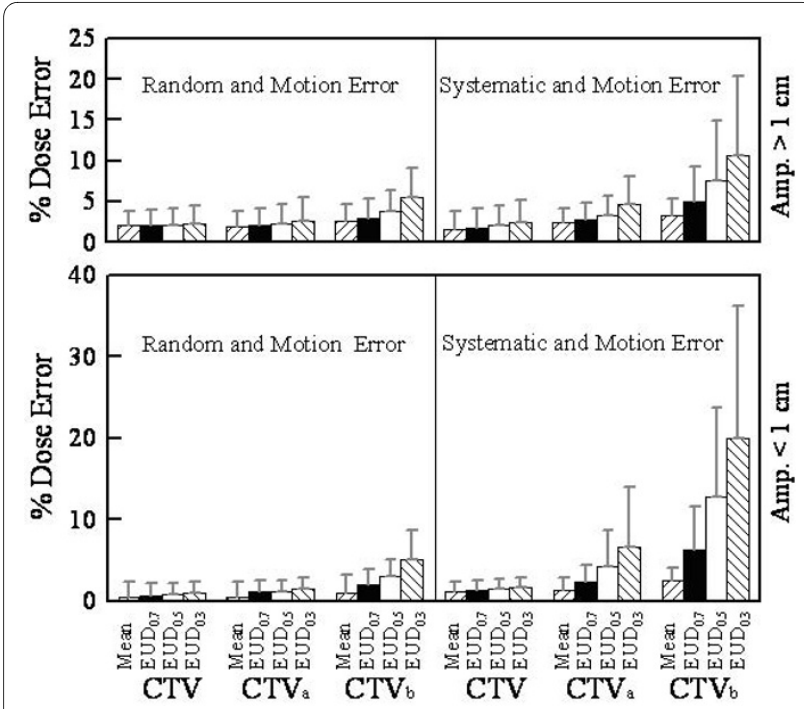

Figure 4 The effects of motion plus random error and motion plus systematic error. The percentage dose errors are shown for patients with motion amplitudes greater than $1 \mathrm{~cm}$ and less than $1 \mathrm{~cm}$. Data are shown for different margin recipes resulting in different CTVs $\left(C T V, C T V_{a}\right.$, and $\left.C T V_{b}\right)$ and over a variety of different radiosensitivities $\left(\mathrm{SF}_{2}=0.7,0.5\right.$ and 0.3$)$. The effects of motion plus random error and motion plus systemic error are separated in the two graphs. The error bars indicate 1 standard deviation.

motion dominates the geometry impact in the inferior direction only. Figure $5 \mathrm{c}$ and $5 \mathrm{~d}$ show the DVH of the plans with and without different geometry uncertainties for targets with sufficient margins $\left(\mathrm{CTV} \& \mathrm{CTV}_{\mathrm{a}}\right)$ and without sufficient margins $\left(\mathrm{CTV}_{\mathrm{b}}\right.$ \& PTV). The EUD errors from the geometry errors are summarized in table 3 for $\mathrm{SF}_{2}$ of $0.3,0.5$ and 0.7 , respectively. The geometric error impact is insignificant for $\mathrm{CTV}$ and $\mathrm{CTV}_{\mathrm{a}}$. It has a small impact for $\mathrm{CTV}_{\mathrm{b}}$ of sensitive cell type $\left(\mathrm{SF}_{2}\right.$ equal to $0.3)$.

\section{Discussion}

Margin design is critical for the dose received by the tumor. An optimum margin is the aperture that ensures the dose received by target with the least possible amount of irradiation of normal tissue. In this study, three different margin recipes were tested. Both CTV to PTV and $\mathrm{CTV}_{\mathrm{a}}$ to PTV are sufficient to accommodate respiration and setup error. Our T-test results at different end points all indicated that the margin recipe of CTV can be replaced by margin recipe of $\mathrm{CTV}_{\mathrm{a}}$; however, the margin recipe of $\mathrm{CTV}_{\mathrm{b}}$ cannot replace the margin recipe of CTV without any compensation.

The $\mathrm{CTV}_{\mathrm{b}}$ has insufficient margin by $0.5 \mathrm{~cm}$ to $1 \mathrm{~cm}$. For the cases of motion amplitude larger than $1 \mathrm{~cm}$ (table 2), the margin from $\mathrm{CTV}_{\mathrm{b}}$ to PTV is close to the margin with random setup error only. This approximates the case in Ten Haken's study [23] where the motion margin was eliminated. In their study, elimination of motion facilitates dose escalation of about $11 \%$ for the same normal tissue complication probability. However, due to the dose smearing effect, the geometric uncertainty results in an actual escalated BED by approximately $5 \sim 8 \%$, depending upon the radiation sensitivity of the tumor cell. This ignores systematic errors. If systematic errors exist during the treatment, the potential dose escalation would be negated by the geometric delivery inaccuracy. In the group of amplitudes greater than $1 \mathrm{~cm}$ in Figure 4 with combined motion and systematic errors, $\mathrm{CTV}_{\mathrm{b}}$ had a mean $\%(\Delta E U D)$ reduction ranging from $3 \%$ to $11 \%$ and a maximum reduction ranging from $6 \%$ to $20 \%$. These also reflect the fact that systematic error has a serious impact

Table 4: The motion characteristics of the case plan with 4D scheme.

\begin{tabular}{cccc}
\hline & Lt/Rt & AP & Sup/Inf \\
\hline Max. displacement & $1.6 \mathrm{~mm}$ & $5.0 \mathrm{~mm}$ & $13.0 \mathrm{~mm}$ \\
95\%_SM & $-2.5 /-3.3$ & $-1.8 /-1.6$ & $-4.0 /-5.0$ \\
95\%_IM & $+1.0 /-0.4$ & $-2.4 /+3.0$ & $+1.4 /-8.8$ \\
95\%_G & $-1.3 /-3.2$ & $-2.4 /+2.6$ & $-1.9 /-12.6$ \\
& & & EUD $_{\text {SF0.7 }}$ \\
\hline & EUD SF0.3 & EUD $_{\text {SF0.5 }}$ & 1.009 \\
CTV_G & 1.010 & 1.010 & 1.006 \\
CTV__G & 1.006 & 1.006 & 0.986 \\
CTV__G & 0.976 & 0.854 & 0.909 \\
PTV_G & 0.788 & & \\
\hline
\end{tabular}

The change of the $95 \%$ dose position of the dose profiles due to different geometry uncertainties and biological impact (EUD) for different margins are summarized. 

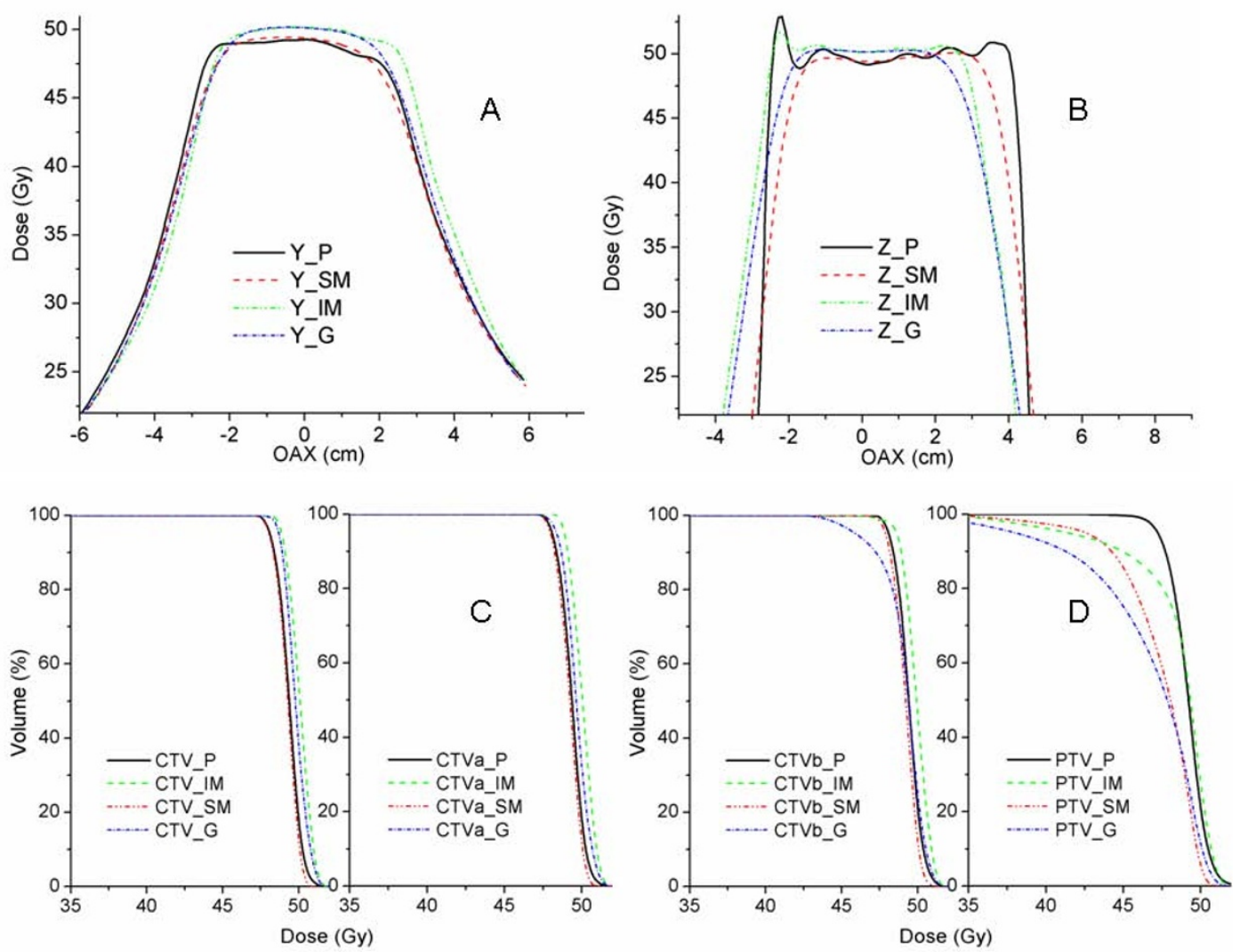

Figure 5 Effects of geometrical uncertainty on the patient with 4D planning. (a) \& (b) display dose profiles (OAX is the off axis distance) of the static plan ("P") and with different geometric impact s("SM", "IM" \&"G") in "Y" and "Z" directions, respectively. (c) \& (d) display the DVH of the plans with

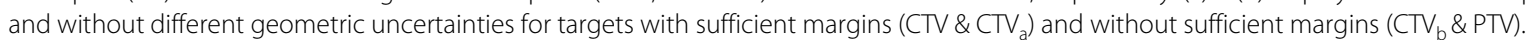

on the equivalent dose received by the target compared to random error. The smaller margin the target has (as in the group of amplitude less than one $\mathrm{cm}$ ), the larger the dosimetric error caused by the $0.5 \mathrm{~cm}$ systematic offset. In practice, elimination of the motion margin should be performed with reliable image guidance techniques (IGRT), and a dose escalation scheme should be considered to compensate for the biologic dose reduction due to the geometric misses during treatment.

We caution that our approach is not universally applicable. Here, we reduced the size of the CTV without altering the size of the PTV. In reality, it is the PTV volume that changes. If applied with constant CTV, PTV with bigger margin would have a larger volume. This will lead to different dose uniformity within the PTV and more dose to normal tissue after the inverse planning of IMRT. The dose gradient between the borders of the PTV will be different, too. Since this study compared the coverage of CTV with and without motion impact after large (25) fractionation IMRT delivery, slight dose non-uniformity (after inverse planning) within the PTV should not affect the results of the CTV coverage in this study. Slight different dose gradient will affect more at PTV and less at CTVs after the dose smeared by motion. The irradiation to normal tissue after motion impact is outside the scope of this study, too. These are issues warrant further study.

The radiation sensitivity of the cell type has a strong influence on the sensitivity of the target to margin reduction. This conclusion can be drawn from the results shown in figures 3 and 4 . Overall, since the dosimetric effect resulting from geometric uncertainties is larger at the target border, where the dose gradient is greater, the radiosensitive tumor cells suffer more from the geometric uncertainties when the margins were insufficient (e.g., $\mathrm{CTV}_{\mathrm{b}}$ in this study). The low dose volume generated by geometric uncertainties has a larger biological impact on more radiation sensitive tumor types. Low dose volumes may be generated during the optimization process. Although the cold spots were outside the CTV, the biological impact could be magnified after the dose blurring by geometrical uncertainties.

Since the impact of the geometric uncertainties is dependent on the tumor cell type, margin reduction should also consider the clinical stage of the tumor and 
the corresponding radiation sensitivity. An example of the implementation is shown in figure 6, which is based on the tumor and tumor cell response to radiation at different clinical stages listed at table 5 . The survival fractions of stage IIb and IIIb HCC are lower than 0.5, which indicate radiation sensitive cell types, so the planning target should use generous margins (eg. $1^{\text {st }}$ and $2^{\text {nd }}$ margin recipe in this study) to avoid the cold spots from geometric misses. For stage IV HCC or HCC with PVT, the radiation response is poorer and the whole CTV is usually too big to spare the normal liver, so a smaller margin ( $2^{\text {nd }}$ and $3^{\text {rd }}$ margin recipe in this study) can be considered. If the motion margin is eliminated, $5 \%$ dose escalation is suggested to compensate for tumor dose loss from geometric uncertainties as shown in figure 4 where the mean $\%(\triangle \mathrm{EUD})$ reduction were between $2 \% \sim 6 \%$ for $\mathrm{CTV}_{\mathrm{b}}$.

This study considers the diaphragm as a surrogate for liver motion and assumed that the liver has rigid motion in Sup/Inf direction only. A case applied 4D planning scheme with 3D deformable organ motion was studied for comparison and is summarized in table 4. In this case, the maximum displacement at RT/LT, AP, and Sup/Inf are $1.6 \mathrm{~mm}, 5 \mathrm{~mm}$ and $13 \mathrm{~mm}$, respectively. The results show that the CTV with sufficient margins (CTV and CTVa) have no dosimetric or biological impact from the geometry uncertainty. When there is insufficient margin (6 $\mathrm{mm}$ in this case), the EUD errors were $2.4 \%, 1.6 \%$ and $1 \%$ for SF2 of 0.3, 0.5, and 0.7, respectively. When no margin was applied (PTV), the EUD errors were 21.2\%, 15.6\% and $9.1 \%$, for SF2 of 0.3, 0.5, and 0.7, respectively. Compared with the results of the rigid body assumption in figures 3 \& 4, where CTV \& CTVa have insignificant dosimetric impacts, CTVb has mean EUD errors typi-

\section{Table 5: Response dose and survival fraction $\left(\mathrm{SF}_{2}\right)$ for different clinical stage of intra-hepatic tumor.}

\section{$\mathrm{SF}_{2}$ for different clinical stage and pathology cell type (Liu, 2005)}

\begin{tabular}{|c|c|c|c|}
\hline & \multicolumn{3}{|c|}{ Clinical stage } \\
\hline & $\mathrm{Ilb}$ & IIllb & IV \\
\hline \multicolumn{4}{|l|}{ Pathology typing } \\
\hline Hepatocellular carcinoma & 0.28 & 0.47 & 0.61 \\
\hline Bile duct epithelial carcinoma & 0.41 & 0.57 & 0.78 \\
\hline \multicolumn{4}{|c|}{ Radiation dose needed for tumor response } \\
\hline & response & \multicolumn{2}{|c|}{ non response } \\
\hline HCC without PVT (Park, 2002) & $50.1 \mathrm{~Gy}$ & \multicolumn{2}{|c|}{$44.3 \mathrm{~Gy}$} \\
\hline HCC with PVT (Kim, 2005) & BED 59.9 Gy & \multicolumn{2}{|c|}{ BED 55.2 Gy } \\
\hline
\end{tabular}

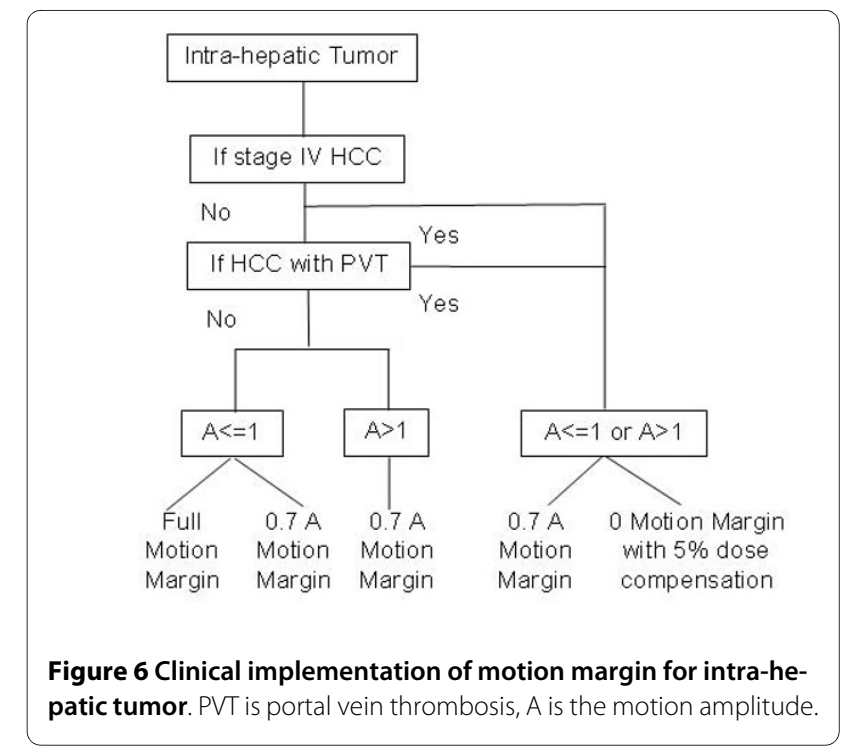

cally between $2 \sim 5 \%$ (with maximum of $8 \%$ ), and PTV has mean EUD errors typically between $6 \sim 12 \%$ ( with maximum 20\%). This result demonstrates a similar conclusion by Brock that deformation is insignificant in affecting the dosimetric coverage of the target and the dose received by normal liver [27]. In other words, this comparison validates the expansion of motion margin design (figure 6) in 3D.

This study only considered $\mathrm{SF}_{2}$ in the implementation of EUD; in addition, the literature regarding the relationship of the $\mathrm{SF}_{2}$ with the cell type of intra-hepatic tumor is very limited. Our method could over simplify the clinical situation. However, the dose dependency of tumor response of different intra-hepatic lesions among different stages of HCC, metastasis lesions, and tumor with or without PVT is very significant. Applying different margin design in clinical practice for a specific patient is not uncommon due to patient's motion amplitude and planning goal of avoiding the RILD of the normal liver. Based on these clinical experiences, this study proposes to combine the laboratory findings, clinical results (compiled as table 5) and the dosimetric effects of geometry uncertainty (summarized as figure 4) in order to design a reasonable and an achievable margin. Of course, further study of the $\mathrm{SF}_{2}$, cell type and the dose response of the intra-hepatic tumor are warranted.

\section{Conclusions}

The biological effects of the geometric uncertainties for intrahepatic lesions depend on margin design and intrinsic radiation sensitivity of the tissue. More radiosensitive tumor cells are more sensitive to the margin size. In the simulation of this study, van Herk's 0.7 A margin is feasible if the inter- and intra- reproducibility of the respiratory motion is also considered. Elimination of the motion 
margin could be beneficial to normal liver sparing with dose escalation, however, the potential dose reduction due to motion blurring on the dose distribution should also be taken into account. The clinical implementation of margin reduction should consider radiosensitivity of the tumor.

\section{Competing interests}

The authors declare that they have no competing interests.

\section{Authors' contributions}

HK designed analyzed and interpreted data. KC participated software programming. WL, CG and SK participated in contouring and helped revise the draft manuscript.

AW, DM, LH and RY participated in study design, data analysis and helped revise the draft manuscript. All authors read and approved the final manuscript.

\section{Author Details}

1Department of Radiation Oncology, Montefiore Medical Center, USA, 2Department Biomedical Engineering and Environmental Sciences, National Tsing Hua University, Taiwan, ${ }^{3}$ Department of Radiation Oncology, University Hospital of Chung-Shan Medical University, Taiwan and ${ }^{4}$ Department of Radiologic Sciences, Thomas Jefferson University, USA

Received: 2 February 2010 Accepted: 3 June 2010

Published: 3 June 2010

\section{References}

1. El-Serag HB, Mason AC: Rising incidence of hepatocellular carcinoma in the United States. NEng/ J Med 1999, 340:745-750

2. Robertson JM, Lawrence TS, Andrews JC, et al:: Long-term results of hepatic artery fluorodeoxyuridine and conformal radiation therapy for primary hepatobiliary cancers. Int J Radiat Oncol Biol Phys 1997, 37:325-330.

3. Dawson LA: Escalated focal liver radiation and concurrent hepatic artery fluorodeoxyuridine for unresectable intrahepatic malignancies. $J$ Clin Oncol 2000, 18(11):2210-8.

4. Park HC, Seong J, Han KH, et al.: Dose-response relationship in local radiotherapy for hepatocellular carcinoma. Int J Radiat Oncol Biol Phys 2002, 54:150-155.

5. Park W, Lim DH, Paik SW, et al.: Local radiotherapy for patients with unresectable hepatocellular carcinoma. Int J Radiat Oncol Biol Phys 2005, 61:1143-50

6. Kim DY, Park W, Lim DH, et al.: Three-dimensional conformal radiotherapy for portal vein thrombosis of hepatocellular carcinoma. Cancer 2005, 103:2419-26.

7. Ryo $T$, Ryuji $M$, Yuji $B$, et al:: Conformal radiation therapy for portal vein tumor thrombosis of hepatocellular carcinoma. Radiother Oncol 2007, 84:266-271

8. West CML, Davison $\mathrm{SE}$, Burt PA, et al:: The intrinsic radiosesitivity of cervical carcinoma: correlations with clinical data. Int J Radiat Oncol Biol Phys 1995, 31:841-846.

9. Stausbol-Gron B, Overgaard J: Relationship between tumor cell in vitro radiosensitivity and clinical outcome after curative radiotherapy for squamous cell carcinoma of the head and neck. Radiother Oncol 1999, 50:47-55.

10. Burnet NG, Jena R, Jefferies $S$ J, et al:: Mathematical modeling of survival of glioblastoma patients suggests a role for radiotherapy dose escalation and predicts poorer outcome after delay to start treatment. Clinical Oncol 2006, 18:93-103.

11. Liu ZZ, Huang WY, Lin JS, et al:: Cell survival curve for primary hepatic carcinoma cells and relationship between SF2 of hepatic carcinoma cells and radiosensitivity. World I Gastroenterol 2005, 11(44):7040-7043.

12. Langen KM, Jones DTL: Organ motion and its management. Int J Radiat Oncol Biol Phys 2001, 50:265-278.

13. Bortfeld T, Jokivarsi K, Goitein M, et al.: Effects of intra-fraction motion on IMRT dose delivery: statistical analysis and simulation. Phys Med Biol 2002, 47:2203-20.
14. Chui CS, Yorke $E$, Hong L: The effects of intra-fraction organ motion on the delivery of intensity-modulated field with a multileaf collimator. Med Phys 2003, 30:1736-46.

15. Kuo HC, Chuang KS, Liu WS, Wu A, Lalonde R: Analysis of the organ motion effects on the effective influences for liver IMRT. Phys Med Biol 2007, 52:4227-44.

16. Goitein M: Organ and tumor motion. Seminars in Radiation Oncology 2004, 14:2-9.

17. Van Herk M: Error and Margins in Radiotherapy. Seminars in Radiation Oncology 2004, 14:52-64.

18. Balter J, Brock K, Lam K, Tatro D, Dawson L, McShan D, Ten Haken R: Evaluating the influence of setup uncertainties on treatment planning for focal liver tumors. Int J Radiat Oncol Biol Phys 2005, 63:610-614.

19. ICRU Report 62: Prescribing, recording, and reporting photon beam therapy (supplement to ICRU Report 50). International Commission on Radiation Units and Measurement, Bethesda, MD; 1999

20. Sdtroom JC, Heijmen BJ: Geometrical uncertainties radiotherapy planning margins and the ICRU-62 report. Radiother Oncol 2002, 64:75-83.

21. Van Herk M, Witte M, Geer JVD, et al:: Biologic and Physical fractionation effects of random geometric errors. Int J Radiat Oncol Biol Phys 2003, 57:1460-1471

22. Mckenzie AL: How should breathing motion be combined with other errors when drawing margins around clinical target volumes? British $J$ Radiology 2000, 73:973-977.

23. Ten Haken RK, Balter JM, Marsh LH, et al: Potential benefits of eliminating planning target volume expansions for patient breathing in the treatment of liver tumors. Int J Radiat Oncol Biol Phys 1997, 38:613-617.

24. Molinelli S, Pooter J, Romero AM, et al:: Simultaneous tumor dose escalation and liver sparing in stereotactic body radiation therapy (SBRT) for liver tumours due to CTV to PTV margin reduction. Radiother Oncol 2008, 87:432-438.

25. Wagman $R$, Yorke E, Ford E, et al:: Respiratory gating for liver tumors: use in dose escalation. Int J Radiat Oncol Biol Phys 2003, 55:659-668.

26. Rietzel E, Liu AK, Doppke KP, Wolfgang JA, Chen AB, Chen GTY, Choi NC: Design of 4D treatment planning target volumes. Int J Radiat Oncol Biol Phys 2006, 66:287-295

27. Brock KK, McShan DL, Ten Haken RK, Hollister SJ, Dawson LA, Balter JM: Inclusion of organ deformation in dose calculation. Med Phys 2003, 30:290-295

28. Balter JM, Lam KL, McGinn CJ, Lawrence TS, Ten Haken RK: Improvement of CT-based treatment-planning models of abdominal targets using static exhale imaging. Int J Radiat Oncol Biol Phys 1998, 41:939-943.

29. Kung JH, Zygmanski $\mathrm{P}$, Choi N, et al:: A method of calculating a lung clinical target volume DVH for IMRT with intrafractional motion. Med Phys 2003, 30:1103-9.

30. McCarter SD, Beckham WA: Evaluation of the validity of a convolution method for incorporating tumor movement and set-up variations into the radiotherapy treatment planning system. Phys Med Biol 2000, 45:923-931.

31. Song W, Battista J, Dyk JV: Limitations of a convolution method for modeling geometric uncertainties in radiation therapy: the radiobiological dose-per-fraction effect. Med Phys 2004, 31:3034-3045.

32. Niemierko A: Reporting and analyzing dose distributions: A concept of equivalent uniform dose. Med Phys 1997, 24:103-109.

33. Kuo HC, Mah D, Chuang KS, Wu A, Hong L, Yaparpalvi R, Spierer M, Kalnicki $S$ : A method incorporating $4 D$ data for evaluating the dosimetric effects of respiratory motion in single arc IMAT. Phys Med Biol 2010, 55:3479-3497.

doi: 10.1186/1748-717X-5-48

Cite this article as: Kuo et al., Biological impact of geometric uncertainties: what margin is needed for intra-hepatic tumors? Radiation Oncology 2010, $\mathbf{5 : 4 8}$ 